\title{
ANGIOLIPOLEIOMIOMA UTERINO: PRESENTACIÓN DE UN CASO Y REVISIÓN DE LA LITERATURA
}

\section{Uterine angiolipoleiomyoma: case presentation and review of the literature}

\author{
Diana Cecilia Poveda-Rojas, $M D^{1}$; Berlly Lucía Díaz-Gómez, $M D^{2}$; \\ Catalina Buriticá-Cifuentes, $\mathrm{MD}^{3}$; Amaury García-Burgos, $\mathrm{MD}^{4}$; \\ Catherine Alvarado-Heine, $M D^{5}$ \\ Recibido: agosto 24/15 - Aceptado: junio 13/16
}

\section{RESUMEN}

Objetivo: presentar el caso de una paciente con angiolipoleiomioma uterino confirmado histopatológicamente y diagnosticado prequirúrgicamente como un teratoma anexial, y realizar una revisión de la literatura respecto al diagnóstico clínico, imagenológico e histopatológico de la entidad.

Materiales y métodos: se presenta el caso de una mujer de 61 años, a quien se le practicó laparotomía por sospecha de un teratoma anexial. En la cirugía se hace diagnóstico de miomatosis uterina y se practica histerectomía abdominal total y salpingooforectomía bilateral. En el estudio histopatológico se encuentran hallazgos compatibles con angiolipoleiomioma uterino. Se realizó una búsqueda en las bases de datos Medline vía PubMed, OVID y en Lilacs, con los términos "angiolipoleiomyoma", "angiolipoleiomyoma uterus", “uterine angiolipoleiomyoma”,

1 Residente de Ginecología y Obstetricia, Universidad Militar Nueva Granada-Hospital Universitario Clínica San Rafael, Bogotá (Colombia).

2 Médica patóloga, Clínica Universitaria Colombia, Departamento de Patología, Bogotá (Colombia). berllyludiaz@gmail.com

3 Médica patóloga, Clínica Reina Sofía, Departamento de Patología, Bogotá (Colombia).

4 Médico Gineco-oncólogo, Clínica Reina Sofía y Clínica Universitaria Colombia, Departamento de Ginecología, Bogotá (Colombia).

5 Médica patóloga, Clínica Colsanitas; directora Laboratorio de Patología, Bogotá (Colombia). "uterine angiolipoleiomyomas" y "angiomyolipoma uterus", se buscaron artículos de revisión, reportes y series de casos en inglés y español publicados desde 1966 hasta mayo de 2016.

Resultados: se encontraron siete artículos, que corresponden a reportes de casos y revisión de la literatura, los cuales describen las principales características en cuanto a presentación clínica, diagnóstico clínico y paraclínicos basándose en pruebas de imagenología y estudios de histopatología.

Conclusión: el angiolipoleiomioma uterino es una entidad benigna muy rara, cuyo diagnóstico diferencial clínico es complejo, ya que simula otras patologías ginecológicas. Es de suma importancia la sospecha clínica prequirúrgica de esta entidad y la posterior confirmación histopatológica.

Palabras clave: angiomiolipoma, leiomioma, teratoma.

\section{ABSTRACT}

Objectives: To present the case of a patient with a uterine angiolipoleiomyoma confirmed by histopathology, diagnosed as adnexal teratoma before surgery, and to conduct a review of the literature regarding clinical, imaging and histopathological diagnosis of this condition. 
Materials and methods: A 61-year old patient taken to laparotomy due a suspected adnexal teratoma. During surgery, uterine myomatosis was diagnosed and total abdominal hysterectomy and bilateral oophorectomy are performed. Findings on histopathology were consistent with uterine angiolipoleiomyoma. A search was conducted in the Medline databases via PubMed, Ovid and in LILACS using the terms "angiolipoleiomyoma", "angiolipoleiomyoma uterus", "uterine angiolipoleiomyoma", "uterine angiolipoleiomyomas" and "angiomyolipoma uterus". The search included review articles and case series and reports published in English and Spanish from 1966 to May 2016.

Results: Seven study articles were found, including case reports and literature reviews describing the main characteristics in terms of clinical presentation, clinical diagnosis and paraclinical tests, based on imaging studies and histopathology. Conclusion: Uterine angiolipoleiomyoma is an extremely rare benign condition that mimics other gynaecological pathologies, making it a difficult clinical differential diagnosis. Preoperative clinical suspicion and postoperative histopathological confirmation are of the utmost importance.

Kew words: Angiomyolipoma, leiomyoma, teratoma.

\section{INTRODUCCIÓN}

El angiolipoleiomioma (ALLM) es un tumor mixto del útero, benigno, de origen mesenquimatoso, que se caracteriza por tener tejido adiposo maduro, músculo liso y un componente vascular, que se encuentra en proporciones variables de rara presentación $(1,2)$. Sus características histológicas lo hacen muy similar al angiomiolipoma renal, razón por la que ha recibido el nombre por algunos autores de angiomiolipoma uterino (AML) $(1,3)$. Su nomenclatura ha sido muy controversial, por lo que ha recibido numerosos nombres como: lipoleiomioma, tumor lipoleiomatoso, tumor mesodérmico mixto benigno, tumor hamartomatoso (4). Pese a los diversos nombres que ha recibido, el nombre aprobado y apropiado para esta entidad es el de ALLM, ya que la mayoría de los AML son de origen renal, reportándose también lugares de localización extrarrenal (hígado, bazo, retroperitoneo, pulmón, piel) que incluyen muy pocos casos documentados a nivel uterino y de tracto genital femenino (5). Los AML son categorizados actualmente dentro de los PEComas (2), lo que hace que se encuentren dentro de sus principales diferencias: a) su origen (origen mesenquimatoso vs. origen de células epiteliales vasculares); b) la asociación de los AML renales con la esclerosis tuberosa (ET) a diferencia de los ALLM que no se asocian a esta; c) su negatividad en la inmunohistoquímica para las características melanocíticas (6).

Dada su incidencia tan baja $(0,06 \%)$ y su presentación clínica inespecífica, con dolor pélvico crónico, sangrado genital anormal, prolapso de órganos pélvicos y sintomatología urinaria, a menudo se confunde con otras patologías ginecológicas, principalmente las de origen anexial (teratomas y masas complejas) y de origen uterino (leiomiomas) $(1,7)$.

Se ha descrito mayor incidencia en mujeres mayores de 40 años, con localización principalmente a nivel del cuerpo del útero, seguido de la localización cervical, con crecimiento intramural o subseroso, sin asociación con la esclerosis tuberosa. Su historia natural no es muy conocida por los pocos casos reportados, su comportamiento es frecuentemente benigno. Respecto al curso clínico pueden presentarse recurrencias si se realiza únicamente miomectomía, por lo cual se ha establecido como elección de tratamiento la histerectomía (2).

$\mathrm{Al}$ ser un tumor raro, que se confunde fácilmente con otras patologías, y de difícil diagnóstico preoperatorio, cobra gran importancia el conocimiento de esta entidad para la práctica diaria, no solo por parte del médico ginecólogo, sino también del clínico tratante y del patólogo; por tanto, con el objetivo de revisar la literatura respecto al diagnóstico clínico, imagenológico e histopatológico de la entidad, se presenta este caso de angiolipoleiomioma. 


\section{REPORTE DE CASO}

Paciente de 61 años, con 5 gestaciones de las cuales 3 fueron partos vaginales, 1 embarazo ectópico y 1 aborto, con última menstruación en el año 2005, que consulta en el primer semestre de 2014 a institución hospitalaria de referencia de una compañía aseguradora privada que atiende pacientes del aseguramiento contributivo y privado en el sistema de salud colombiano, ubicada en Bogotá (Colombia), por cuadro clínico de dolor pélvico crónico, de presentación ocasional, sin otra sintomatología asociada. El examen físico se reporta dentro de límites normales, sin signos de irritación peritoneal ni masas palpables. Antecedentes de relevancia: carcinoma papilar de tiroides manejado con tiroidectomía total y laparotomía por embarazo ectópico. Linfoma cutáneo T estadio Ib manejado con terapia con radiación ultravioleta A y psoraleno (PUVA-terapia) (8).

Dentro de los estudios iniciales se realiza una ecografía transvaginal (figura 1) que muestra una imagen dependiente de ovario izquierdo, redondeada, heterogénea, predominantemente sólida, con áreas quísticas y contenido ecogénico que sugiere grasa, con medidas de $75 \times 44 \times 70 \mathrm{~mm}$, con un volumen de $122 \mathrm{cc}$; por lo que se concluye un

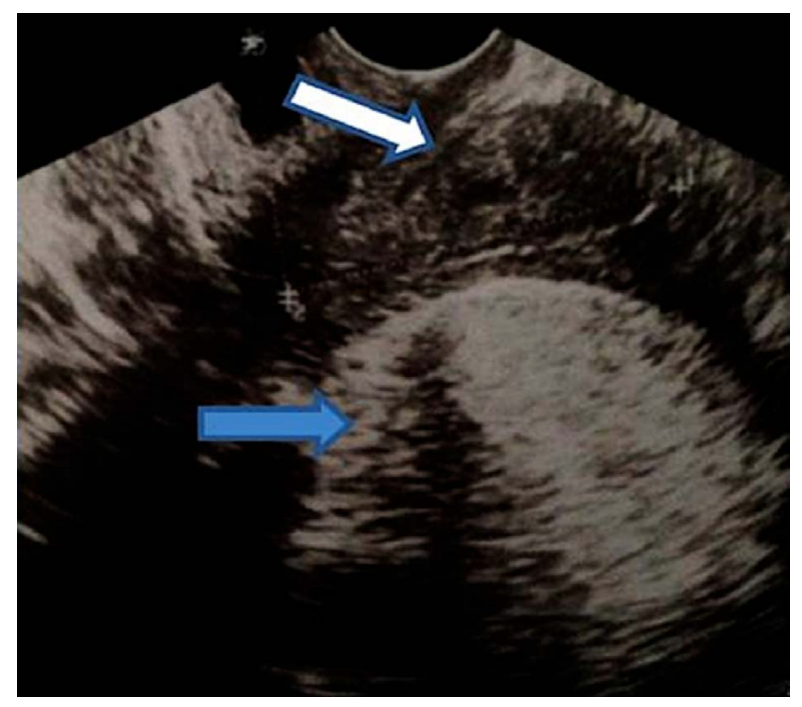

Figura 1. Ecografía con imagen redondeada heterogénea, con contenido ecogénico, que sugiere grasa (flecha azul) anterior al cuerpo uterino (flecha blanca) teratoma quístico izquierdo. Llaman la atención el tamaño y las características de la lesión, por lo que se realiza TAC de abdomen total (figura 2) en la que se observa una masa en la línea media anterior al útero, de contornos bien definidos, con componente mixto, densidad de tejido blando y adiposo, sin otras masas ni adenomegalias. Se concluye una masa anexial compleja compatible con teratoma.

La paciente es programada para laparotomía exploratoria con diagnóstico prequirúrgico de quiste anexial complejo, con sospecha de teratoma; durante el procedimiento quirúrgico elegido inicialmente se reconocen múltiples miomas subserosos e intramurales, y anexos atróficos, por lo que se realiza histerectomía abdominal total y salpingooforectomía bilateral. El procedimiento se realiza sin complicaciones.

En el servicio de patología se recibe producto de histerectomía total, con sus anexos, con un peso de $179 \mathrm{~g}$; la serosa es lisa y con una gran masa en la cara anterior que deforma el cuerpo uterino. Al corte, el canal endocervical y la cavidad endometrial se encuentran conservadas, y en el miometrio se reconoce una masa bien definida subserosa de $6 \times 6 \times 4,5 \mathrm{~cm}$, blanquecina, de aspecto arremolinado, con áreas amarillentas de tejido de aspecto

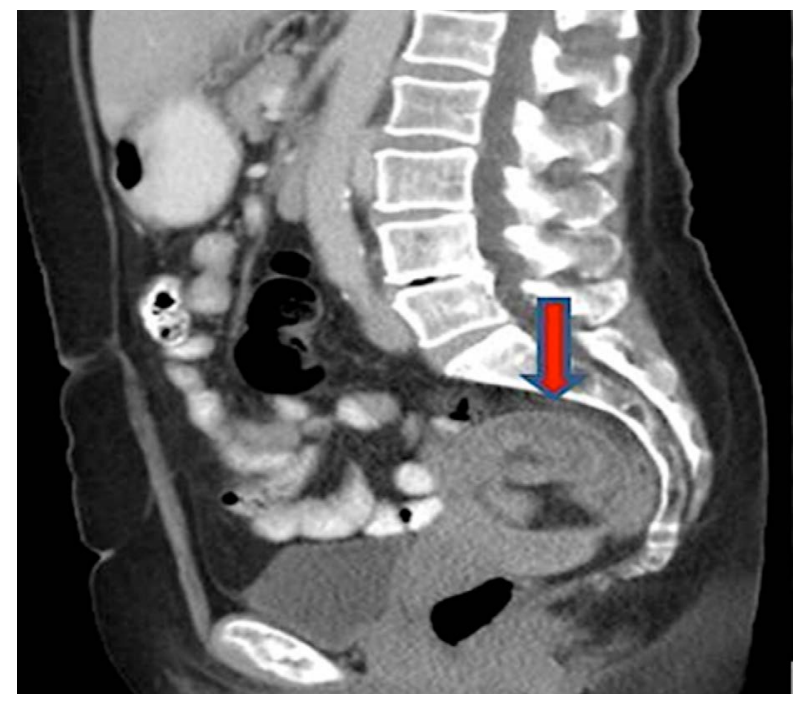

Figura 2. Masa en la línea media anterior al útero, de contornos bien definidos, aspecto heterogéneo, con densidad de grasa en su interior (flecha roja) 


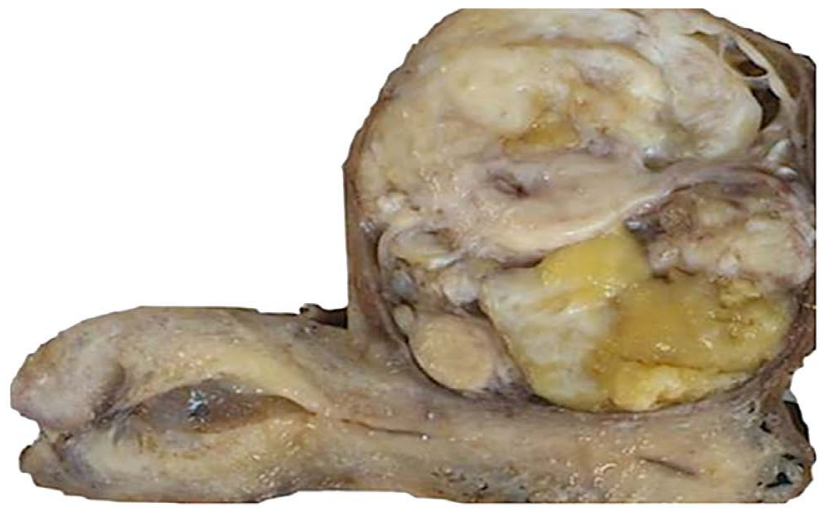

Figura 3. Corte longitudinal. Cavidad endometrial colapsada, miometrio de la cara anterior con masa subserosa bien definida, de $6 \times 6 \times 4,5 \mathrm{~cm}$, blanquecina, arremolinada, con áreas amarillentas de tejido de aspecto adiposo

adiposo (figura 3). Los anexos se encuentran con cambio de atrofia sin lesiones.

A los cortes microscópicos (figura 4) se evidencia una lesión tumoral benigna de origen mesenquimal, constituida por células musculares lisas maduras, las cuales se disponen en un patrón arremolinado, asociado a proliferación de adipocitos maduros, los cuales se ubican formando lóbulos separados por septos de tejido fibroconectivo con numerosos vasos sanguíneos de diferentes tamaños. El conteo mitótico es menor de 1 en 10 campos de alto poder (CAP). No hay pleomorfismo, ni necrosis coagulativa. Los estudios de inmunohistoquímica muestran negatividad del HMB45 (figura 5) y un índice de proliferación celular medido con Ki67 menor al 1\% (figura 6).
Posteriormente se realiza control a la paciente donde se evalúa diagnóstico prequirúrgico, hallazgos intraoperatorios y diagnóstico final informado por patología; la paciente evoluciona asintomática, por lo que se cierra el caso sin complicaciones.

Aspectos éticos. Se solicitó autorización tanto a la paciente como al Comité de Ética en Investigacion de la Fundación Universitaria Sanitas para la publicación de las fotos. Se garantizó la confidencialidad de la información y la identificación de la paciente para proteger sus derechos.

\section{MATERIALES Y MÉTODOS}

Se realizó una búsqueda de la literatura en Medline vía PubMed y BVS, usando los términos: "angiolipoleiomyoma", "angiolipoleiomyoma uterus", “uterine angiolipoleiomyoma”, "uterine angiolipoleiomyomas" y "angimyolipoma uterus". Se incluyeron estudios que trataran sobre el diagnóstico y las características clínicas, imagenológicas e histopatológicas de esta entidad. Se buscaron artículos de revisión, reportes y series de casos sin restricción de idioma, desde 1996 a mayo de 2016. Se excluyeron publicaciones de angiolipoleiomioma cutáneo y renal, al igual que angiolipoleiomioma en animales (caninos).

\section{RESULTADOS}

Se obtuvieron 56 títulos sobre angiolipoleiomioma (ALLM) y angiomiolipoma (AML) uterino en las bases de datos de búsqueda, de los cuales se excluyeron 48 artículos debido a que 47 de ellos eran de
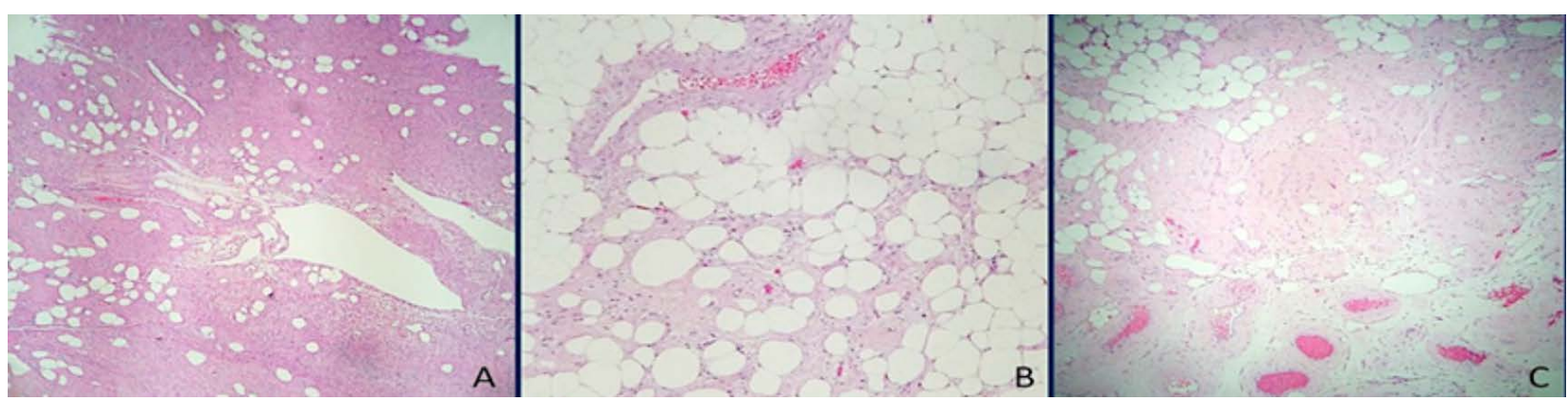

Figura 4. Cortes microscópicos. Células musculares lisas maduras, en un patrón arremolinado, asociado a proliferación de adipocitos maduros, que forman lóbulos separados por septos fibroconectivos con numerosos vasos sanguíneos 


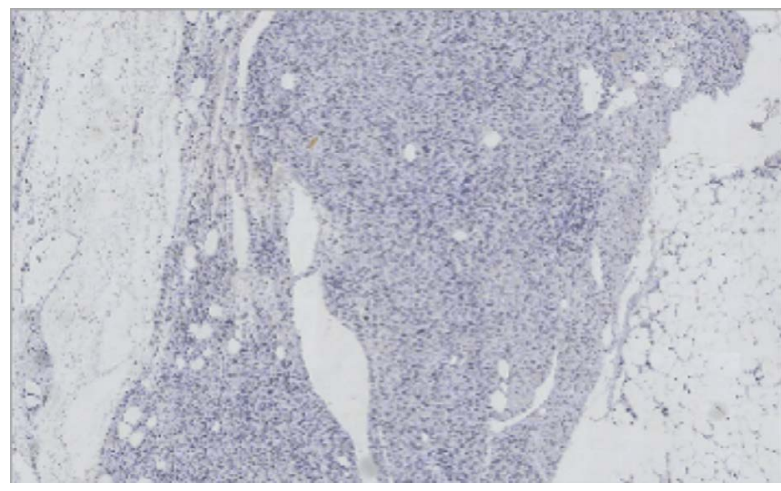

Figura 5. HMB-45: negativo

localizaciones extrauterinas (renales, pulmonares, hepáticas y cutáneas) y 1 era un estudio en animales. Se incluyeron 4 artículos que tratan de angiolipoleiomioma (ALLM) uterino, 3 de angiomiolipoma (AML) uterino y uno de tumor lipoleiomiomatoso uterino. Todos corresponden a reportes de caso (1-9) con revisión de la literatura publicados entre 1989 y 2013. Son publicaciones en inglés $(1,3-7,9)$ y eslovaco (2). Estos fueron hechos en Europa (2, 3, 6), Asia $(4,5,9)$ y Norteamérica $(1,7)$.

Presentación clínica. El angiolipoleiomioma generalmente es asintomático, por lo que el diagnóstico es un hallazgo incidental encontrado por imágenes (1). Cuando se presentan los síntomas, estos son similares a los leiomiomas clásicos, tales como dolor abdominal crónico, hemorragia uterina anormal, polaquiuria, incontinencia y prolapso de los órganos pélvicos $(1,7,9)$.

Presentación imagenológica. Dentro de sus principales características ecográficas se encuentra su aspecto como masas irregulares, brillantes, con alta ecogenicidad, correspondiente a tejido lipomatoso, asociado a ecogenicidad, sin sombra, visto en los vasos sanguíneos del tejido lipomatoso que lo hacen simular teratomas benignos (1). Se pueden extender desde el miometrio hasta la subserosa (1) y presentar pequeñas áreas anecoicas dentro de la masa (7).

\section{Diagnóstico histopatológico}

Anatomía macroscópica. En su aspecto macroscópico el tamaño va desde 2 hasta $16 \mathrm{~cm}$, con un promedio

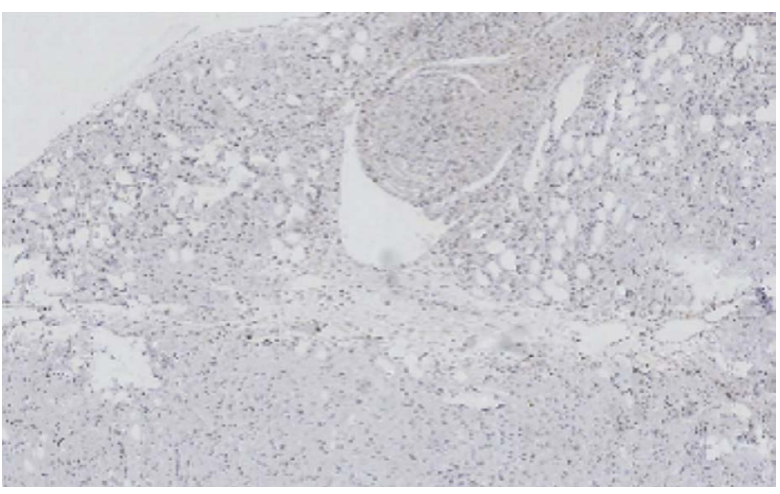

Figura 6. Índice de proliferación celular medido con Ki67

de 8,4 cm $(1,8)$; usualmente son bien definidos, con una pseudocápsula, pero en ocasiones pueden presentar un crecimiento infiltrativo (1). Su consistencia varía entre elástica o firme, lo cual depende de la proporción de cada uno de los componentes. Al corte en la superficie pueden tener coloración gris clara, amarillenta o rosada, que varía en apariencia, siendo muy raro encontrar necrosis o hemorragia $(1,6,9)$.

Estudio microscópico. En las coloraciones de rutina se evidencian fascículos de células de músculo liso, entremezcladas con tejido adiposo y fibroconectivo. Pueden organizarse en un patrón arremolinado imitando leiomiomas. El tejido adiposo es maduro, con citoplasma claro y núcleos periféricos. Se pueden observar ocasionales lipoblastos. Los vasos sanguíneos generalmente son tortuosos y similares a pequeñas o medianas arterias, con paredes fusionadas imperceptiblemente con las capas fibromusculares que rodean el tejido del estroma, con red capilar bien desarrollada. La proporción en los componentes no ha sido determinada a la hora de establecer criterios diagnósticos de ALLM; sin embargo, en ninguno de los tres componentes es usual ver atipia o mitosis $(1,4,9)$.

Estudios de inmunohistoquímica han evidenciado que las células musculares demuestran inmunorreactividad citoplásmica contra la actina y desmina, y todos los ALLM son negativos con HMB-45, marcador de células epiteliales melanocíticas; lo contrario a los AML renales $(1,2,4,6)$. 


\section{CONCLUSIONES}

Es poca la literatura encontrada respecto a ALLM, donde juegan un papel importante las ayudas imagenológicas en el diagnóstico prequirúrgico, siendo el criterio definitivo el estudio histopatológico para su confirmación, tal como se realizó en este caso.

\section{AGRADECIMIENTOS}

Agradecemos a los docentes del departamento de Patología de la Clínica Universitaria Colombia, a la Clínica Reina Sofía, al Comité de Ética en Investigación de la Fundación Universitaria Sanitas y al Departamento de Imágenes Diagnósticas de la Clínica Colsanitas, por su abordaje diagnóstico en este caso y por la colaboración en la realización de este reporte.

\section{REFERENCIAS}

1. Ren R, Wu HH. Pathologic Quiz Case A 40-YearOld Woman With an Unusual Uterine Tumor. 2004;128:31-2.

2. Kajo K, Zúbor P, Krivus S, Danko J. Angiolipoleiomyoma of the uterus. Case report and literature review. Ceska Gynekol. 2010;75:54-6.

3. Laffargue F, Giacalone PL, Charpin C, Lachard A. Uterine angiomyolipoma associated with pregnancy. Gynecol Oncol. 1993;50:357-60.
4. Yaegashi H, Moriya T, Soeda S, Yonemoto Y, Nagura H, Sasano H. Uterine angiomyolipoma: case report and review of the literature. Pathol Int. 2001;51:896-901.

5. Lee SJ, Yoo JY, Yoo SH, Seo YH, Yoon JH. Uterine angiomyolipoma with metastasis in a woman with tuberous sclerosis: a case report. Eur J Gynaecol Oncol. 2013;34:339-42.

6. Cil AP, Haberal A, Hucumenoglu S, Kovalak EE, Gunes M. Angiomyolipoma of the uterus associated with tuberous sclerosis: case report and review of the literature. Gynecol Oncol. 2004;94:593-6.

7. Braun HL, Wheelock JB, Amaker BH, Seeds JW. Sonographic evaluation of a uterine angiolipoleiomyoma. J Clin Ultrasound. 2002;30:241-4.

8. Olsen EA, Hodak E, Anderson T, Carter JB, Henderson M, Cooper K, et al. Guidelines for phototherapy of mycosis fungoides and Sézary syndrome: A consensus statement of the United States Cutaneous Lymphoma Consortium. J Am Acad Dermatol. 2016;74:27-58. doi: 10.1016/j. jaad.2015.09.033. Epub 2015 Nov 4.

9. Shintaku M. Lipoleiomyomatous tumors of the uterus: A heterogeneous group? Histopathological study of five cases. Pathol Int. 1996;46:498-502. 